\title{
Shear Stress Prediction Using FEA-ANN Hybrid Modeling Of Eicher 11.10 Chassis Frame
}

\author{
Tushar M Patel ${ }^{1}$, Dr N M Bhatt ${ }^{2}$ \\ ${ }^{1}$ Research Scholar: Mewar University, Gangrar, Chittorgarh, Rajasthan, India. \\ ${ }^{2}$ Director: Gandhinagr Institute of Technology, Moti Bhoyan, Gandhinagar, Gujarat, India.
}

\begin{abstract}
The chassis serves as a backbone for supporting the body and different parts of the automobile. It should be rigid enough to withstand the shock, twist, vibration and other stresses. Along with strength (Shear Stress), an important consideration in chassis design is to have adequate bending stiffness (Deflection). The main objective of the research is to develop an ANN model for shear stress prediction. The chassis frame is made of two side members joined with a series of cross members. The number of cross members, their locations, cross-section and the sizes of the side and the cross members becomes the design variables. The chassis frame model is to be developed in Solid works and analyzed using Ansys. Since the no. of parameters and levels are more, the probable models are too many. The weight reduction of the sidebar is achieved by changing the Parameters using the orthogonal array. Then FEA is performed on those models. ANN model is prepared using the results of FEA. For the ANN modeling, the standard back-propagation algorithm is found to be the best choice for training the model. A multi- layer perception network is used for non-linear mapping between the input and output parameters. This model can save material used, production cost and time.
\end{abstract}

Keywords: Optimization, Chassis frame, FE analysis, FEA-ANN hybrid modeling, Weight reduction

\section{INTRODUCTION}

In automotive type vehicles, the frame is considered to be the foundation or "Backbone". The frame in conjunction with the vehicle suspensions, axles, wheels and tires make up the principal load-carrying components of a vehicle. The frame and other components don't only carry the weight of the vehicle, but its payload as well. In addition to the load carrying function, the frame and suspension also transfer the forces from the axles to the vehicle structure. This includes the forces of brake torque reaction as well as the drive forces that propel or move the vehicle. The frame acts as a foundation or base for the body structure of vehicles, the axles with their suspensions and the engine/transmission package. The frame must be rigid enough to support or carry all the loads and forces that the vehicle is subjected to in operation. A frame must also be flexible enough to handle shock loads and the twists, bends, sway and sag that it encounters under different road or load conditions. A frame that is too rigid is most likely to fail even under normal operations. Ideally the frame should be able to flex under different situations, while being able to return to its original shape when loads or forces are removed.

According to European Commission of Research \& Innovation in transport, the reduction of fuel consumption and $\mathrm{CO} 2$ emissions is one of the most important challenges facing the automotive industry. One way to reduce consumption is by reducing a weight of the vehicle. Thus, the project goal is to provide the basis to save millions of tonnes of fuel and carbon dioxide due to significantly reduced vehicle weight. About onethird of a passenger car's total fuel consumption directly depends on its weight. A weight reduction of $100 \mathrm{~kg}$ represents a fuel savings of between $0.3-0.5$ liters for every $100 \mathrm{~km}$ driven according to industry estimates [Pratelli (1966)].

The main objective of the project is to Prepare ANN model to predict Shear Stress for Eicher 11.10 chassis frame. As the chassis frame is analyzed using the finite element techniques, appropriate model of the frame is to be developed. The weight reduction is achieved by changing the Parameters (Size Optimization) of the sidebar and cross bar. Then FEA is performed on those models to get the best model. Since the numbers and levels of parameters are more, the probable models are too many. So, to select optimum parameters among them large numbers of modelling and analysis work is involved which consumes more time. To overcome this problem, Design of Experiment technique will be used along with FEA and than ANN model will be prepared.

\section{LITERATURE REVIEW}

Structural optimization using computational tools has become a major research field in recent years. Methods commonly used in structural analysis and optimization may demand considerable computational cost, depending on the problem complexity. Among these ANN may be combined with classical analysis, to reduce the computational effort without affecting the final solution quality. Bourquina et al. (1998) used Artificial Neural Networks (ANN) methodology to analyze experimental data from a tabulating study and compared both 
graphically and numerically to classical modelling techniques. Javadi et al. (2003) founded that Finite element method has been widely used as a powerful tool in the analysis of engineering problems. In this numerical analysis, the behaviour of the actual material is approximated to that of an idealized material that deforms in accordance with some constitutive relationships. Spina et al. (2006) optimized injection moulded product by using an integrated environment. The approach implemented take advantages of the Finite Element (FE) Analysis to simulate component fabrication and investigate the main causes of defects. A FE model was initially designed and then reinforced by integrating Artificial Neural Network to predict main filling and packing results and Particle Swarm Approach to optimize injection moulding process parameters automatic. This research has confirmed that the evaluation of the FE simulation results through the Artificial Neural Network system was an efficient method for the assessment of the influence of process parameter variation on part manufacturability, suggesting possible adjustments to improve part quality. Saltan et al. (2007) introduced a new concept of integrating artificial neural networks (ANN) and finite element method (FEM) in modelling the unbound material properties of the sub - base layer in flexible pavements. Benardos et al. (2007) have been adopted the multitude of different approaches in order to deal with this problem which has investigated all aspects of the ANN modelling procedure, from training data collection and pre/post-processing to elaborate training schemes and algorithms. Cardozo et al. (2011) presented the formulation and implementation of a computational code to optimize manufactured complex laminated structures with a relatively low computational cost by combining the Finite Element Method (FEM) for structural analysis, Genetic Algorithms (GA) for structural optimization and ANN to approximate the finite element solutions.

\section{MATERIAL OF MODEL}

The material for the chassis is defined ST 52 which is widely used material for the chassis. The material properties are as shown in Table 1.

Table 1: Material properties of chassis (Tech, 2003)

\begin{tabular}{|l|l|}
\hline Material & ST 52 \\
\hline Modulus of Elasticity E & $2 \times 105 \mathrm{MPa}$ \\
\hline Poisson's Ratio & 0.3 \\
\hline Tensile Strength & $520 \mathrm{MPa}$ \\
\hline Yield Strength & $360 \mathrm{MPa}$ \\
\hline
\end{tabular}

\section{METHODOLOGY}

As an important subject in the statistical design of experiments, the Taguchi method is a collection of mathematical and statistical techniques useful for the parametric optimization and analysis of problems in which a response of interest is influenced by several variables and the objective is to optimize this response.

Taguchi method is used to examine the relationship between a response and a set of quantitative experimental variables or factors.

\section{Steps for the Experiment:}

- Formulation of the problem - the success of any experiment is dependent on a full understanding of the nature of the problem.

- Selection of the output performance characteristics most relevant to the problem.

- Selection of parameters.

- Selection of factor levels.

- Design of an appropriate Orthogonal Array (OA).

- To Perform FEA with appropriate set of parameters.

- Statistical analysis and interpretation of experimental results.

- The neural network design and development was done using MATLAB R2008a for the results obtained by Taguchi method.

- The predicted ANN shear stress data is compared with actual data obtained by experiment performed on the basis of Taguchi method for training, validation and testing.

Flow chart of the experiment is given in Fig. 1. 


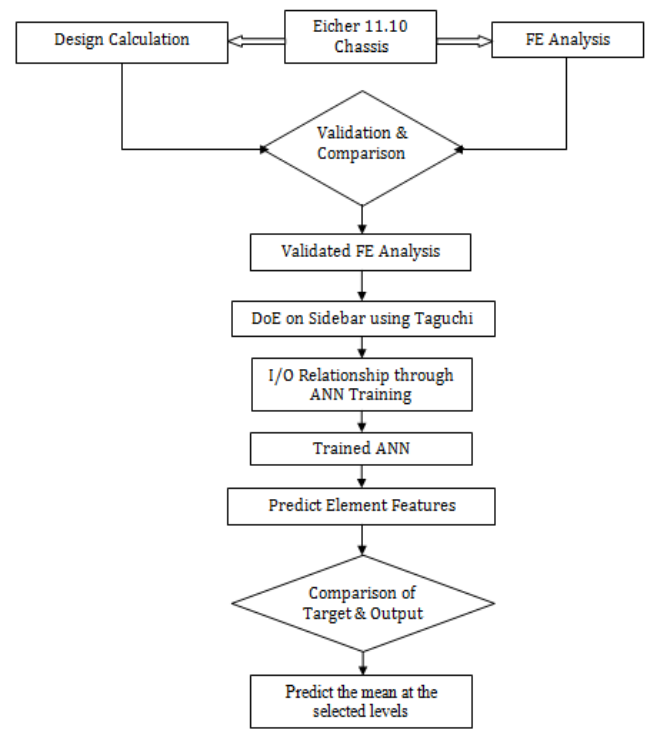

Fig. 1 Flow chart of Experiment

\section{EXPERIMENTAL METHOD}

Experiments are planned according to Taguchi's L25 orthogonal array for web, upper flange and lower flange as shown in Fig.2. It has 25 rows corresponding to the number of tests with 5 columns at five levels and 3 parameters as shown in Table 2. This orthogonal array is chosen due to its capability to check the interactions among factors.

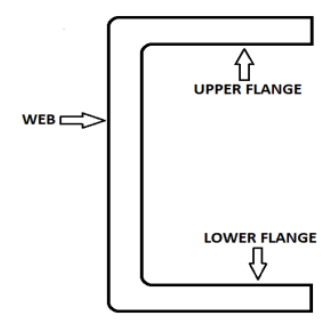

Fig. $2 \mathrm{C}$ channel

Table 2. Factors and their levels

\begin{tabular}{|l|l|l|l|l|l|}
\hline \multicolumn{1}{|c|}{ Factor } & Level 1 & Level 2 & Level 3 & Level 4 & Level 5 \\
\hline Thickness of Web (mm) & 3 & 4 & 5 & 6 & 7 \\
\hline Thickness of Upper flange (mm) & 3 & 4 & 5 & 6 & 7 \\
\hline Thickness of Lower flange (mm) & 3 & 4 & 5 & 6 & 7 \\
\hline
\end{tabular}

For finding out the optimum thickness of web, upper flange and lower flange the value of shear stress, deflection and weight is measured using ANSYS. Series of analysis is conducted to obtain the optimum weight for allowable stress and deflection condition. Taguchi method is being applied to select the control factor levels (thickness of web, upper flange and lower flange) to come up with an optimal response value (weight, shear stress and deflection).

Taguchi design experiments using specially constructed tables known as "orthogonal arrays" (OA). The use of these tables makes the design of experiments very easy and consistent.

From the Table 3 it is identified that minimum shear stress value $70.491 \mathrm{MPa}$ and minimum deflection value $2.7419 \mathrm{~mm}$ are obtained at the experiment number 25 having values of thickness of the web, thickness of upper flange and thickness of lower flange $7 \mathrm{~mm}, 7 \mathrm{~mm}$ and $6 \mathrm{~mm}$ respectively. 
Table 3. Experimental Results Table

\begin{tabular}{|c|c|c|c|c|c|c|}
\hline $\begin{array}{l}\text { Sr. } \\
\text { No. }\end{array}$ & $\begin{array}{c}\text { Thickness of } \\
\text { web } \\
(\mathbf{m m})\end{array}$ & $\begin{array}{c}\text { Thickness of } \\
\text { upper flange } \\
(\mathrm{mm})\end{array}$ & $\begin{array}{c}\text { Thickness of } \\
\text { lower flange } \\
(\mathrm{mm})\end{array}$ & $\begin{array}{c}\text { Weight } \\
\text { (Kg) }\end{array}$ & $\begin{array}{c}\text { Shear stress } \\
\text { (N/mm2) }\end{array}$ & $\begin{array}{l}\text { Deflection } \\
\text { (mm) }\end{array}$ \\
\hline 1 & 3 & 3 & 3 & 222.2 & 150.45 & 5.0147 \\
\hline 2 & 3 & 4 & 4 & 237 & 120.55 & 4.5912 \\
\hline 3 & 3 & 5 & 5 & 250.5 & 130.24 & 3.7103 \\
\hline 4 & 3 & 6 & 6 & 263.8 & 114.8 & 3.4359 \\
\hline 5 & 3 & 7 & 7 & 277.14 & 98.638 & 3.1076 \\
\hline 6 & 4 & 3 & 4 & 248.32 & 129.73 & 4.6805 \\
\hline 7 & 4 & 4 & 5 & 262.39 & 127.19 & 4.2384 \\
\hline 8 & 4 & 5 & 6 & 275.5 & 118.78 & 3.6374 \\
\hline 9 & 4 & 6 & 7 & 289 & 109.686 & 3.4383 \\
\hline 10 & 4 & 7 & 3 & 279.4 & 123.99 & 3.6753 \\
\hline 11 & 5 & 3 & 5 & 279.44 & 123.3 & 3.9242 \\
\hline 12 & 5 & 4 & 6 & 288.4 & 115.77 & 3.4818 \\
\hline 13 & 5 & 5 & 7 & 301.76 & 110.39 & 3.1643 \\
\hline 14 & 5 & 6 & 3 & 281.69 & 122.35 & 3.437 \\
\hline 15 & 5 & 7 & 4 & 295.07 & 119.43 & 3.2619 \\
\hline 16 & 6 & 3 & 6 & 301.475 & 112.22 & 3.4309 \\
\hline 17 & 6 & 4 & 7 & 314.66 & 99.647 & 3.0272 \\
\hline 18 & 6 & 5 & 3 & 294.88 & 111.44 & 3.3299 \\
\hline 19 & 6 & 6 & 4 & 308.06 & 104.21 & 3.0888 \\
\hline 20 & 6 & 7 & 5 & 321.25 & 102.69 & 2.9097 \\
\hline 21 & 7 & 3 & 7 & 327.75 & 107.2 & 3.1379 \\
\hline 22 & 7 & 4 & 3 & 308.27 & 109.91 & 3.3075 \\
\hline 23 & 7 & 5 & 4 & 321.26 & 103.59 & 3.0497 \\
\hline 24 & 7 & 6 & 5 & 334.25 & 98.796 & 2.8711 \\
\hline 25 & 7 & 7 & 6 & 347.23 & 70.489 & 2.7419 \\
\hline
\end{tabular}

\section{ANN APPROACH For SHEAR STRESS PREDICTION}

Literature reviews also show that ANN models have better prediction capability than the regression models. So ANN models are also created for shear stress prediction. This section describes pre processes, model design and training, model simulation and post processes in the generation of ANN prediction models.

Before applying inputs and outputs for ANN training, data have to be converted into a range of 0 to 1 or -1 to 1 i.e. data should be normalized for ANN training. An equation no. 1 was used for data normalization which ranges the data to $[0,1]$. Normalized and randomized result table is shown in Table 4.

$$
\mathrm{x}_{\mathrm{n}}=\frac{\mathrm{x}-\mathrm{x}_{\min }}{\mathrm{x}_{\max }-\mathrm{x}_{\min }}
$$

Where,

$\mathrm{xn}=$ Normalized Value of Variable $\mathrm{x}$

$\mathrm{x}=$ Value of Variable $\mathrm{x}$

$\mathrm{xmin}=$ Minimum Value of variable $\mathrm{x}$

$\mathrm{xmax}=$ Maximum Value of Variable $\mathrm{x}$

All 25 experimental data sets are divided for training, validation and testing. Using GUI in Neural Network Toolbox in MATLAB, different network configuration with different number of hidden neurons is trained and their performance is checked. There are 17 data sets are used for training, 4 data sets for validation and 4 data sets for testing. It is clear that more data sets in training reduces processing time in ANN learning and improves the generalization capability of models, so large number of data sets are used to train the models. Attempts have been made to study the network performance with a different number of hidden neurons. A network is constructed each of them is trained separately, and the best network is selected based on the accuracy of the predictions in the testing phase. 
Table 4. Normalized Experiment Result Table

\begin{tabular}{|c|c|c|c|c|c|}
\hline \multirow{2}{*}{$\begin{array}{c}\text { Number of } \\
\text { Experiment } \\
\text { No. }\end{array}$} & \multicolumn{3}{|c|}{ Factors } & \multirow{2}{*}{$\begin{array}{c}\text { Mean Shear } \\
\text { Stress } \\
(\mathrm{N} / \mathbf{m m} 2)\end{array}$} & \multirow[t]{2}{*}{ Remarks } \\
\hline & $\begin{array}{c}\text { Thickness of } \\
\text { web } \\
(\mathrm{mm})\end{array}$ & $\begin{array}{c}\text { Thickness of } \\
\text { upper flange } \\
\text { (mm) }\end{array}$ & $\begin{array}{c}\text { Thickness of } \\
\text { lower flange } \\
(\mathrm{mm})\end{array}$ & & \\
\hline 1 & 0 & 0 & 0 & 1 & Training \\
\hline 2 & 0 & 0.25 & 0.25 & 0.623557 & Validation \\
\hline 3 & 0 & 0.5 & 0.5 & 0.747245 & Training \\
\hline 4 & 0 & 0.75 & 0.75 & 0.554147 & Validation \\
\hline 5 & 0 & 1 & 1 & 0.35198 & Testing \\
\hline 6 & 0.25 & 0 & 0.25 & 0.740867 & Testing \\
\hline 7 & 0.25 & 0.25 & 0.5 & 0.709101 & Training \\
\hline 8 & 0.25 & 0.5 & 0.75 & 0.603922 & Testing \\
\hline 9 & 0.25 & 0.75 & 1 & 0.490189 & Training \\
\hline 10 & 0.25 & 1 & 0 & 0.660804 & Training \\
\hline 11 & 0.5 & 0 & 0.5 & 0.652451 & Training \\
\hline 12 & 0.5 & 0.25 & 0.75 & 0.566278 & Training \\
\hline 13 & 0.5 & 0.5 & 1 & 0.498993 & Training \\
\hline 14 & 0.5 & 0.75 & 0 & 0.64857 & Testing \\
\hline 15 & 0.5 & 1 & 0.25 & 0.612051 & Training \\
\hline 16 & 0.75 & 0 & 0.75 & 0.52188 & Training \\
\hline 17 & 0.75 & 0.25 & 1 & 0.364637 & Training \\
\hline 18 & 0.75 & 0.5 & 0 & 0.512125 & Validation \\
\hline 19 & 0.75 & 0.75 & 0.25 & 0.421704 & Training \\
\hline 20 & 0.75 & 1 & 0.5 & 0.402694 & Validation \\
\hline 21 & 1 & 0 & 1 & 0.449098 & Training \\
\hline 22 & 1 & 0.25 & 0 & 0.49299 & Training \\
\hline 23 & 1 & 0.5 & 0.25 & 0.41395 & Training \\
\hline 24 & 1 & 0.75 & 0.5 & 0.353969 & Training \\
\hline 25 & 1 & 1 & 0.75 & 0 & Training \\
\hline
\end{tabular}

\section{NEURAL NeTWORK DESIGN}

A feed-forward neural network with back propagation is used. The network consists of three layers. The first layer, which is the input layer, is triggered using the sigmoid activation function whereas the second layer is hidden layer and third layer is the output layer which is triggered using the linear activation function as shown in Fig.3. A network of two transfer function, where the first transfer function is signed and the second transfer function is linear, can be trained to approximate any function.

The network is trained using a suitable supervised learning algorithm, in this case, the LevenbergMarquardt algorithm. In the case of supervised learning, the network is presented with both the input data and the target data called the training set. The network is adjusted based on comparison of the output and target values until the outputs match the targets.

After the data have been normalized, input data files and targets data files are created for training purpose. These input data files include file for training, validation and testing which contains input data sets in random order. Target data files include targets (normalized measured shear stress values respectively of input data sets) for training, validation and testing data sets. The work in this paper included a function approximation or prediction problem that required the final error to be reduced to a very small value.

ANN model is created, trained and simulated, and model used 3 layers - one input layer, one hidden layer and one output layer. Numbers of neurons in the input and output layer were fixed and they were 4 and 1 respectively. In this study one hidden layer with 20 neurons were used. In model tansig transfer function was used in between input layer and output layer, whereas purelin transfer function was used in between hidden layer and output layer.

The work in this paper included a function approximation or prediction problem that required the final error to be reduced to a very small value and, in general, the networks were of moderate size.

Fig. 3 suggests how this model is designated. This designation covers various properties of the ANN model created. It covers types of training algorithm used, number of neurons in the hidden layer, transfer function used in between input and hidden layer, and in between hidden and output layer. 


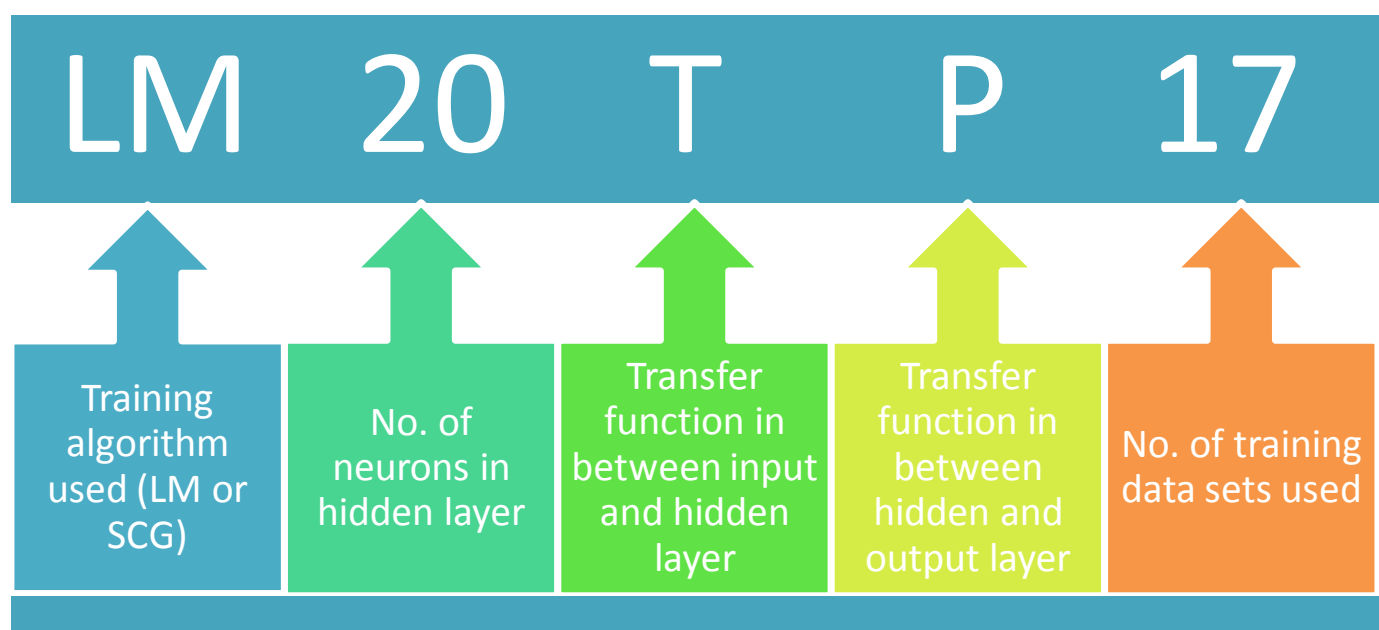

Fig. 3 ANN Model designation

Fig. 4 shows general view of LM20TP model, whereas Figure 5 shows simplified view of LM20TP Model. Fig. 6 shows Abbreviated view of LM20TP Model in MATLAB window and Fig. 7 shows neural network toolbox model creation and training window of LM20TP model. It is back propagation model type used LM training algorithm which has 20 neurons in hidden layer, MSE performance function, tansig and purelin transfer function is used in between input and hidden layer, and in between hidden and output layer respectively.

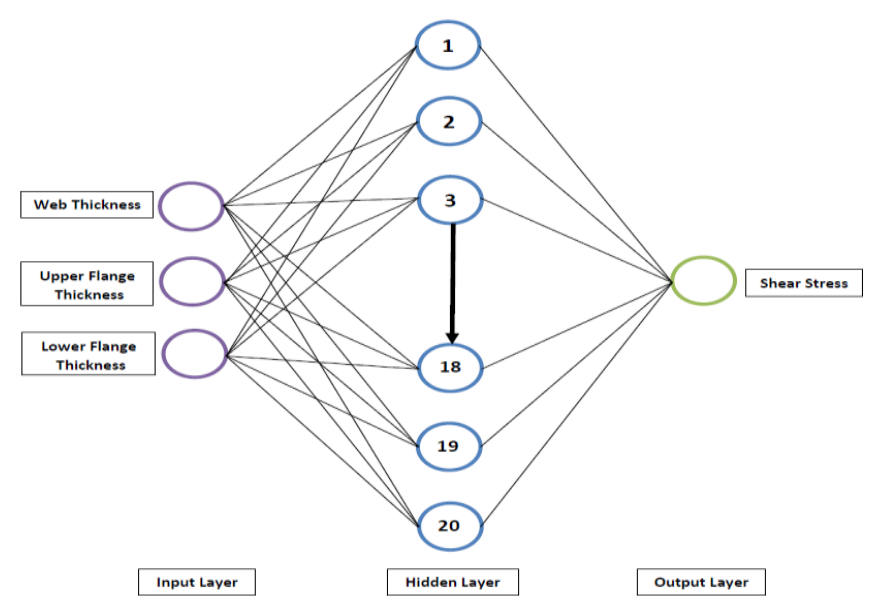

Fig. 4 General view of LM20TP Model view with three layers

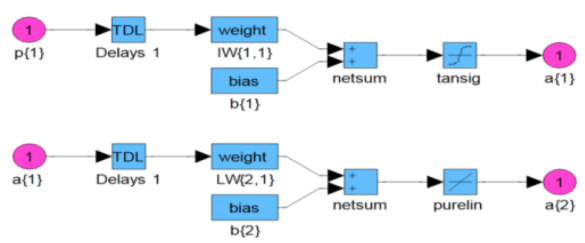

Fig.5 Simplified View of LM20TP Model

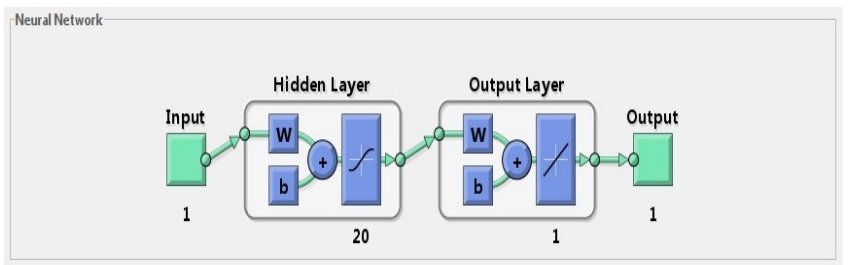

Fig. 6 Abbreviated view of LM20TP Model in MATLAB window 


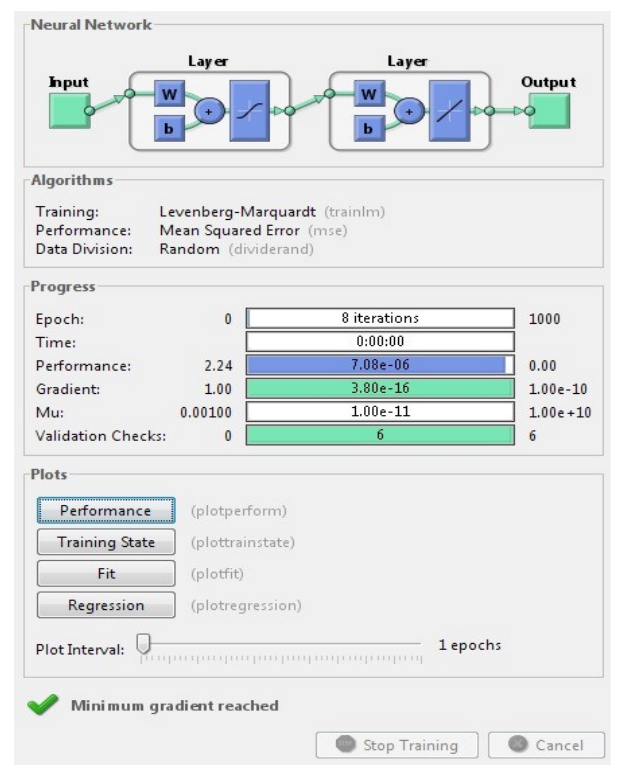

Fig.7 LM20TP model Training

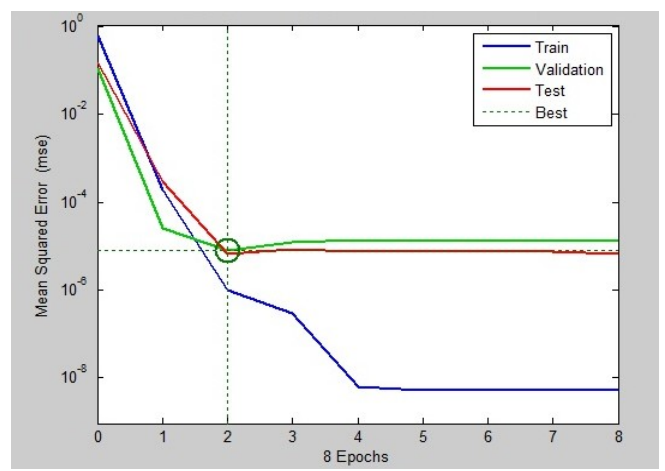

Fig. 8 LM20TP Model Training Performance Graph (retrained)

Fig. 8 shows retrained performance (MSE) graph of LM20TP model, created during its training. The training stopped after 8 epochs because the validation error increased. It is a useful diagnostic tool to plot the training, validation, and test errors to check the progress of training.

The result here is reasonable, because the test set error and the validation set error have similar characteristics, and it doesn't appear that any significance over fitting has occurred. After initial training of LM20TP model, it is retrained for 8 epochs and performance MSE is obtained 7.07702e-006 in training.

The methodology selected to check the prediction and generalization capability of models are discussed in following subsections.

\section{RESULT AND DISCUSSION}

In order to understand whether an ANN is making good predictions, test data that have never been presented to the network are used and the results are checked at this stage. The statistical methods of root mean square error (RMSE), the coefficient of multiple determination (R2) values have been used for making comparisons. These values are determined by the following equations:

$$
\begin{aligned}
& \text { RMSE }=\left[\frac{1}{n} \sum_{j=1}^{n}\left|a_{j}-p_{j}\right|^{2}\right]^{\frac{1}{2}} \\
& R^{2}=1-\left[\frac{\sum_{j=1}^{n}\left(a_{j}-p_{j}\right)^{2}}{\sum_{j=1}^{n}\left(p_{j}\right)^{2}}\right]
\end{aligned}
$$

MATLAB tool is used to check the errors generated in prediction model, after trained and simulated ANN results are exported in to MATLAB work space. All 25 results are checked for two types of error terms 
after training and simulation result obtained. Summarized result is shown in Table 5 which shows errors in training, validation and testing separately. This model is performing well in shear stress prediction in training, validation and testing.

Table 5. Training, validation, and testing data sets used for ANN analysis

\begin{tabular}{|c|c|c|c|c|c|c|c|c|}
\hline $\begin{array}{l}\text { Sr. } \\
\text { No. }\end{array}$ & $\begin{array}{c}\text { Number of } \\
\text { Experiment } \\
\text { No. }\end{array}$ & Remarks & $\begin{array}{c}\text { Exp. Shear } \\
\text { Stress } \\
\text { N/mm2 }\end{array}$ & $\begin{array}{c}\text { Predicted } \\
\text { Shear Stress } \\
\text { N/mm2 }\end{array}$ & $\begin{array}{l}\text { Error in } \\
\text { microns } \\
\text { N/mm2 }\end{array}$ & $\begin{array}{l}\text { Percentage } \\
\text { error } \\
(\%)\end{array}$ & RMSE & $\mathbf{R}^{2}$ \\
\hline 1 & 1 & Training & 150.45 & 150.4462012 & 0.0037988 & 0.002525 & \multirow{17}{*}{ 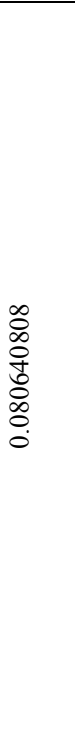 } & \multirow{17}{*}{ 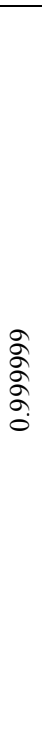 } \\
\hline 2 & 3 & Training & 130.24 & 130.2392119 & 0.0007881 & 0.000605 & & \\
\hline 3 & 7 & Training & 127.19 & 127.2000280 & -0.0100280 & -0.00788 & & \\
\hline 4 & 9 & Training & 109.686 & 109.7618983 & -0.0758760 & -0.06918 & & \\
\hline 5 & 10 & Training & 123.3282 & 123.1904419 & 0.1377881 & 0.111725 & & \\
\hline 6 & 11 & Training & 122.6603 & 122.8004478 & -0.1401198 & -0.11423 & & \\
\hline 7 & 12 & Training & 115.77 & 115.7825674 & -0.0125673 & -0.01086 & & \\
\hline 8 & 13 & Training & 110.39 & 110.2926134 & 0.0973866 & 0.088221 & & \\
\hline 9 & 15 & Training & 119.43 & 119.4178197 & 0.0121803 & 0.010199 & & \\
\hline 10 & 16 & Training & 112.22 & 112.2325117 & -0.0125117 & -0.01115 & & \\
\hline 11 & 17 & Training & 99.647 & 99.7430357 & -0.0960357 & -0.09638 & & \\
\hline 12 & 19 & Training & 104.21 & 104.3327181 & -0.1227181 & -0.11776 & & \\
\hline 13 & 21 & Training & 106.4004 & 106.3701793 & 0.0302307 & 0.028412 & & \\
\hline 14 & 22 & Training & 109.91 & 109.9497197 & -0.0397197 & -0.03614 & & \\
\hline 15 & 23 & Training & 103.59 & 103.4569676 & 0.1330324 & 0.128422 & & \\
\hline 16 & 24 & Training & 98.794 & 98.6861225 & 0.1078774 & 0.109194 & & \\
\hline 17 & 25 & Training & 70.491 & 70.4923276 & -0.0013276 & -0.00188 & & \\
\hline 18 & 2 & Validatio & 120.35 & 120.2250550 & 0.1249441 & 0.103817 & \multirow{4}{*}{ 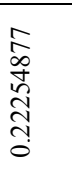 } & \multirow{4}{*}{$\begin{array}{l}\text { ڤे } \\
\text { ूे } \\
\text { ूे }\end{array}$} \\
\hline 19 & 4 & Validatio & 114.8 & 115.0912190 & -0.2912189 & -0.25368 & & \\
\hline 20 & 18 & Validatio & 111.44 & 111.1480989 & 0.2919010 & 0.261936 & & \\
\hline 21 & 20 & Validatio & 102.69 & 102.5782589 & 0.1117410 & 0.108814 & & \\
\hline 22 & 5 & Testing & 98.635 & 98.4804689 & 0.1545310 & 0.15667 & \multirow{4}{*}{ 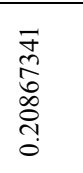 } & \multirow{4}{*}{ ลे. } \\
\hline 23 & 6 & Testing & 129.73 & 129.6027592 & 0.1272407 & 0.098081 & & \\
\hline 24 & 8 & Testing & 118.78 & 119.0550213 & -0.2750213 & -0.23154 & & \\
\hline 25 & 14 & Testing & 122.35 & 122.5918090 & -0.2418089 & -0.19764 & & \\
\hline
\end{tabular}
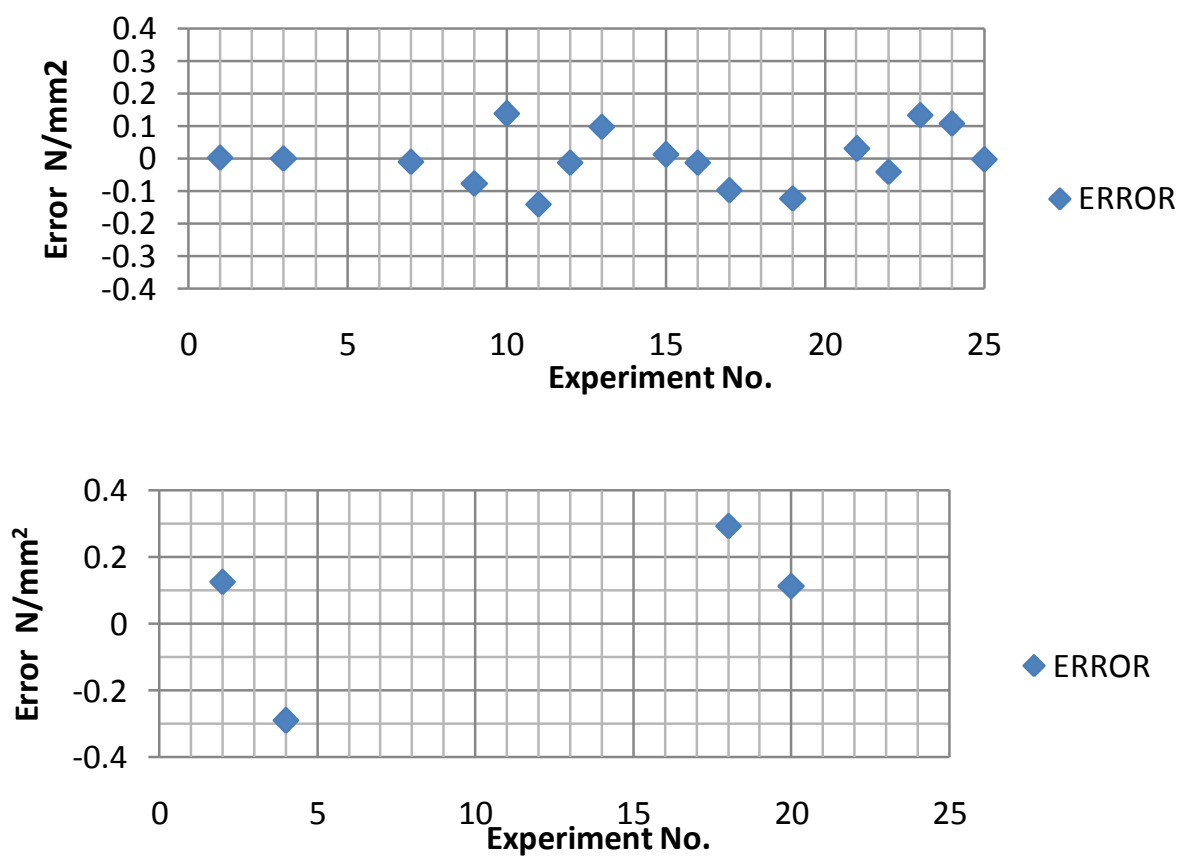


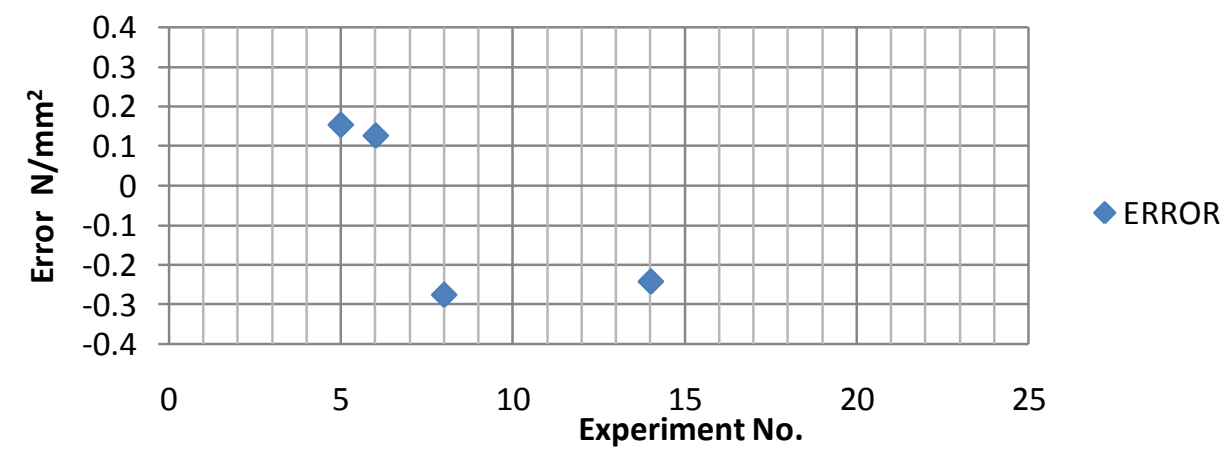

Fig. 9 LM20TP_17 Model Prediction Error in a) Training, b) Validation and c) Testing

Prediction errors in training, validation and testing for LM20TP_17 model are shown in Fig. 9.

\subsection{LM20TM ANN MODEL WEIGHTS}

ANN model is trained by changing and storing proper weights in interconnection links between neurons lying in various layers. These weight values are the responsible parameters which give prediction capability to trained ANN models. Weighs in connection links between input and hidden neurons, and neurons in hidden and output layer for LM20TM are shown in Table 6.

Table 6. Weights in Connections of LM20TP Model

\begin{tabular}{|l|l|l|l|l|l|}
\hline \multicolumn{7}{|l|}{ Weights in between input and hidden layer } \\
\hline N1 & -27.947894706910 & N8 & 27.9944739805161 & N15 & 28.0000003664583 \\
\hline N2 & 28.0003929114959 & N9 & -27.9999874385459 & N16 & -27.9998714512525 \\
\hline N3 & 28.0001011887511 & N10 & -28.0002654351772 & N17 & -27.9779520300813 \\
\hline N4 & 27.9806880993791 & N11 & 27.9992574507772 & N18 & -28.0198157037156 \\
\hline N5 & 28.0027316851348 & N12 & -28.0500621236064 & N19 & 28.1129708786271 \\
\hline N6 & 28.0828299896305 & N13 & -28.0104597096640 & N20 & -27.7044929478809 \\
\hline N7 & -28.1421151592075 & N14 & -28.0003853886688 & & \\
\hline \multicolumn{7}{|l|l|}{ Weights in between hidden and output layer } & N15 & 0.040890430598805 \\
\hline N1 & 0.486050251024637 & N8 & -0.363477923137418 & N16 & -0.082638579223104 \\
\hline N2 & 0.21457304180076 & N9 & 0.21381263524907154 & N17 & -0.050935624005417 \\
\hline N3 & 0.57841023283917 & N10 & -0.713608729841253 & N18 & -0.049493458487956 \\
\hline N4 & -0.4960126870861 & N11 & 0.0285710464271873 & N19 & 0.050325583033594 \\
\hline N5 & 0.16577965829076 & N12 & -0.0549572038243511 & N20 & -0.158876869320821 \\
\hline N6 & 0.048501072175407 & N13 & -0.0872221902484017 & & \\
\hline N7 & -0.08779580329491 & N14 & -0.00904712728418743 & \\
\hline
\end{tabular}

\subsection{LINEAR REgRESSION FITTING OF LM20TP MODEL}

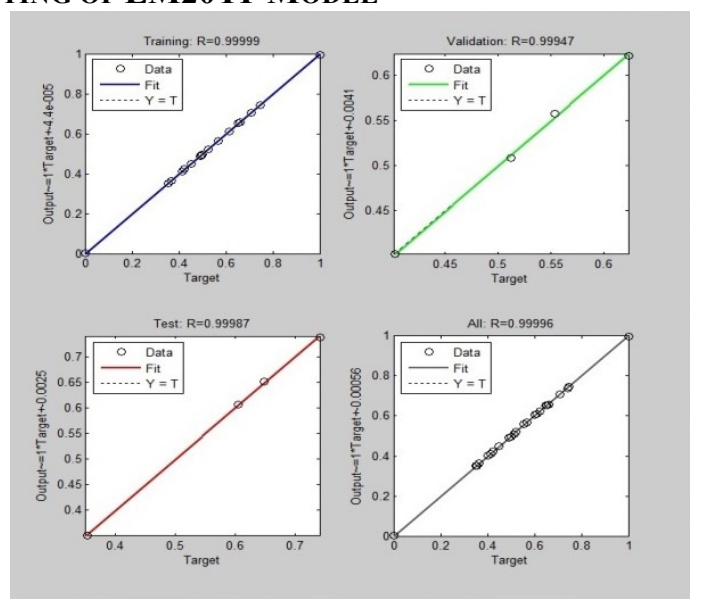

Fig.10 LM20TP Model Linear Fitting in Training, Validation and Testing

The performance of a trained network can be measured to some extent by the errors on the training, validation and test sets, but it is often useful to investigate the network response in more detail. One option is to 
perform a regression analysis between the network response and the corresponding targets. The routine postreg is designed to perform this analysis.

The network output and the corresponding targets are passed to postreg. It returns three parameters. The first two, $\mathrm{m}$ and $\mathrm{b}$, correspond to the slope and the $\mathrm{y}$-intercept of the best linear regression relating targets to network outputs. If it has a perfect fit (outputs exactly equal to the targets), the slope would be 1 , and the yintercept would be 0 . The third variable returned by postreg is the correlation coefficient (R-value) between the outputs and targets. It is a measure of how well the variation in the output is explained by the targets. If this number is equal to 1 , then there is a perfect correlation between targets and outputs. It is performed between the network outputs and the supplied targets for training, validation and testing. Fig. 10 shows the linear regression for training and testing of LM20TP model respectively with three parameters $\mathrm{m}, \mathrm{b}$ and R. Graphs and respective parameters show that LM20TP model linearly closely fits with the supplied target values. This indicates LM20TP model is well suited for shear stress prediction with high accuracy.

\subsection{Comparison of Experimental Results and Predicted LM20TP ANN Results}

\section{Training}

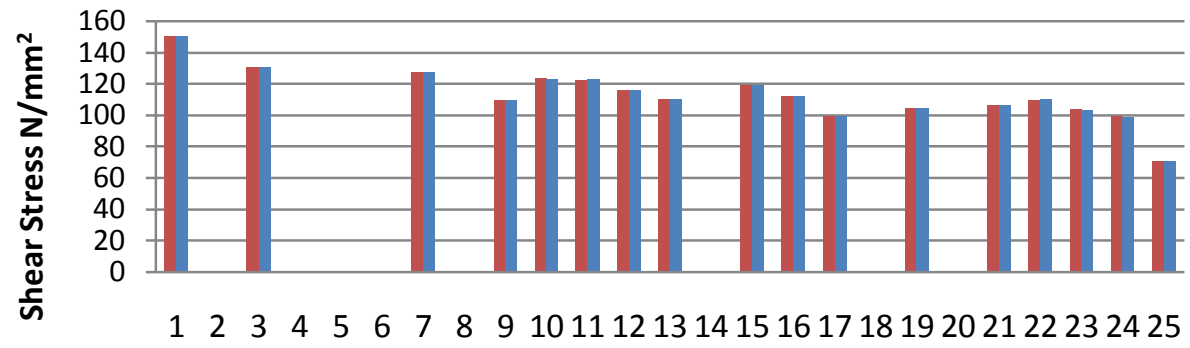

Experiment No.

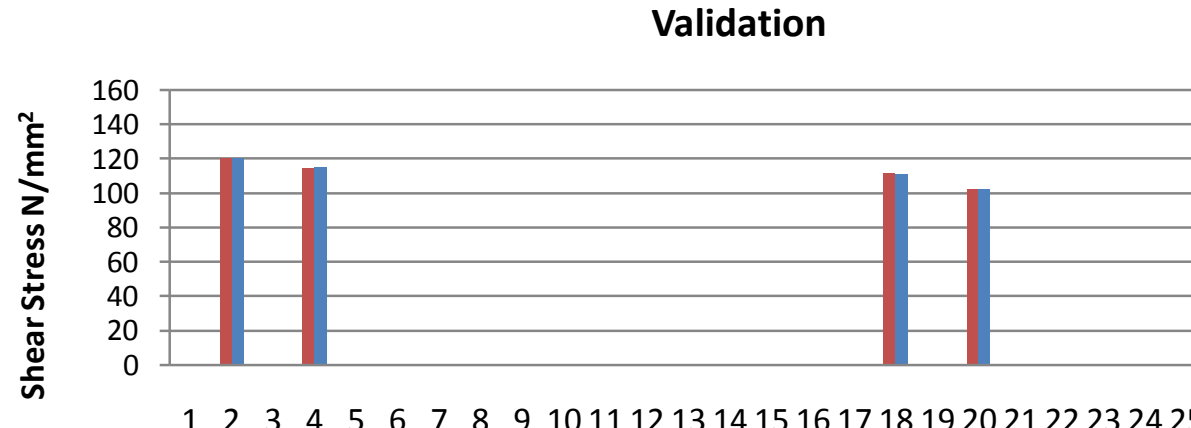

Experiment No.

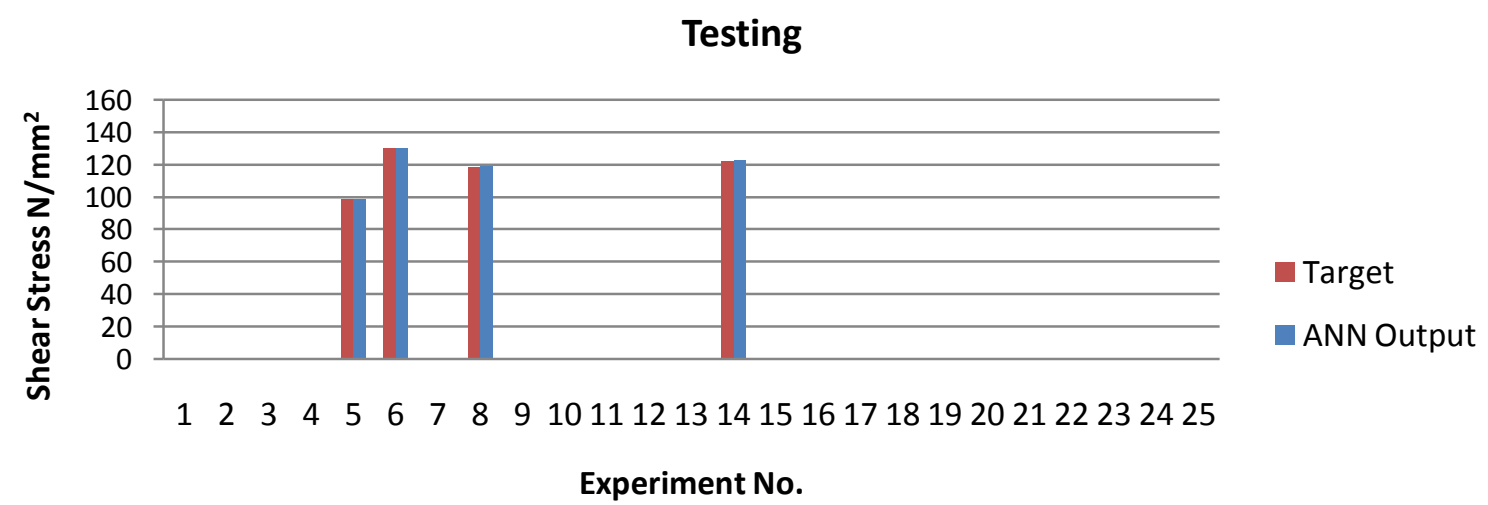

Fig.11 Actual Vs ANN predicted result in Training, Testing and Validation 
Shear stress predicted by selecting LM20TP model is compared with the actual target in training, Validation and in testing is shown in Fig. 11. The comparison is shown by different colours and markers. It is clear from the graph that ANN predicted results are very close to actual targets. It also concludes that the LM20TP ANN model is much better than the linear regression model in prediction capability.

\section{CONCLUSION}

The present investigation aimed at optimization of Shear stress for Eicher 11.10 chassis frame. This optimization is carried out by developing shear stress models based on L25 orthogonal array in Taguchi optimization technique. An ANN based model is developed to predict shear stress of Eicher 11.10 chassis frame using Back propagation network. Levenberg-Marquardt algorithm is used to train the neural network. The ANN model for shear stress prediction draws the following conclusions.

- It is proved that each predicted shear stress values of the ANN are very close to the experimental results.

- It is also concluded that the ANN may be used as a good alternative for the analysis of the effects of chassis frame parameters on the shear stress.

\section{Journal Papers:}

\section{References}

[1]. Bourquin, J., Schmidli, H., van Hoogevest, P., \& Leuenberger, H. (1998). Comparison of artificial neural networks (ANN) with classical modelling techniques using different experimental designs and data from a galenical study on a solid dosage form. European journal of pharmaceutical sciences, 6 (4), 287-300.

[2]. Javadi, A. A., Tan, T. P., \& Zhang, M. (2003). Neural network for constitutive modelling in finite element analysis. Computer Assisted Mechanics and Engineering Sciences, 10 (4), 523-530.

[3]. Spina, R. (2006). Optimization of injection moulded parts by using ANN-PSO approach. Journal of Achievements in Materials and Manufacturing Engineering,15 (1-2), 146-152.

[4]. Saltan, M., \& Sezgin, H. (2007). Hybrid neural network and finite element modeling of sub-base layer material properties in flexible pavements. Materials \& design, 28(5), 1725-1730.

[5]. Benardos, P. G., \& Vosniakos, G. C. (2007). Optimizing feedforward artificial neural network architecture. Engineering Applications of Artificial Intelligence, 20 (3), 365-382.

[6]. Cardozo, S. D., Gomes, H., \& Awruch, A. (2011). Optimization of laminated composite plates and shells using genetic algorithms, neural networks and finite elements. Latin American Journal of Solids and Structures, 8 (4), 413-427.

\section{Books:}

[7]. Pratelli, Antonio. \& Brebbia, C. A. (1966). Urban Transport Seventeen, Part XVII (Southampton, UK: WIT Press).

[8]. Tech, P. S. G. (2003). Design Data Book. Kalaikathir Achchagam, Coimbatore. 